\title{
Yogic Tradition and Well-being
}

\author{
Dr. Jyotsna Agrawal, Associate Professor, Department of Clinical Psychology, \\ National Institute of Mental Health and Neuro Sciences (NIMHANS), Bangalore, INDIA \\ Email id: jyotsna.agl@gmail.com
}

\begin{abstract}
This paper delineates the concept of well-being in modern psychological literature, the variety of pathways studied and its relationship with meaning and spirituality. It further discusses the cultural criticism of modern study of well-being and makes a case for insights from non-western cultures to be included in the mainstream literature. It further discusses the various types of happiness as well as pathways towards them, as discussed in the Indian tradition. These ideas have been substantiated by empirical research and some of the results have been presented in this chapter. It further presents a yogic psycho-spiritual model, based on thematic analysis of core yogic texts, which may be utilized in future for well-being interventions.
\end{abstract}

\section{BACKGROUND}

\section{Happiness and Well-being}

The word happiness is commonly used to designate something intricate and ambiguous, one of those ideas, which humanity has intentionally left vague so that each individual might interpret it in their own way (Bergson, 2006).

Happiness or well-being are the most popular terms used in psychological literature to denote a good life. These terms are often used as rough synonyms, without following any clear distinction. Even the various definitions of happiness sometimes have vague or even contradictory meanings. The dictionary meaning of the noun 'happiness' is, feeling pleasure, contentment, satisfaction or being fortunate. In general, it is associated with feeling positive emotions (of short duration) or mood (for comparatively longer duration). On further exploration, it has been found that personal meanings of happiness go beyond a pleasant emotional state, and also includes one's thoughts and judgements about how one's life is going ( $\mathrm{Lu}, 2001)$. This is similar to the meaning of the term subjective well-being (SWB), which focuses on the subjective experiences of the people, and captures two components; affective (frequency of positive and negative affect) and cognitive (judgment about being satisfied with life). The subjective wellbeing is also known as hedonic wellbeing, and emotional wellbeing, which we will discuss later. In addition, the average positive affect during a specific time period is known as chronic happiness (Lyubomirsky, Sheldon and Schkade, 2005).

Therefore, one's retrospective summary judgment regarding both one's mood and satisfaction, during a certain time frame is captured by SWB. Averaging of all such momentary judgments made at several different times, during a certain period (such as the past two, six or twelve months), also contributes to SWB. In research, this requires measuring and quantifying reported levels of positive affect (PA), negative affect (NA) and life satisfaction (LS). In the literature, some 
researchers have treated the terms happiness and SWB interchangeably and others have used them in specific contexts. While happiness may be used to denote lay concepts, subjective well-being is considered a more scientific term. For example, happiness term is often used when studying the personal meanings, lay beliefs and folk understanding of a good, happy and livable life, and also for exploring what people value in general.

An individual's personal model of well-being, their on-going reflection on and interpretation of where one is now, in contrast to where one was, its modifications, all influence their well-being judgements (Rubinstein, 1989). One's life circumstances may influence their mood states, which in turn may provide them with a hedonic summary of recent life experiences (reactive index) and inform them about progress and prospects in important life domains (prospective index), thereby influencing their life satisfaction (Mood-mediation model; Robinson, 2000).

Other models of well-being, which are more objective in their approach have also been proposed, the foremost being psychological and social wellbeing. Ryff and Keyes (1995) gave a six-factor model of psychological wellbeing; the factors being - autonomy, environmental mastery, personal growth, positive relations with others, purpose in life and self-acceptance. Later Keyes (1998) also developed the social well-being model, which has five components; social acceptance, social actualization, social coherence, social contribution, and social integration. The research on the 3 kinds of well-being (subjective, psychological and social) have expanded over the last few decades, as scientific community has responded to the interest of people at large (Kim-Prieto, Diener, Tamir, Scollon and Diener, 2005) by increasing their efforts to understand the correlates of happiness and wellbeing (Myers and Diener, 1995; Linley and Joseph, 2004).

\section{The varied influences on well-being: Genetics, life context and intentional activities}

For a long time, the scientific community considered happiness and SWB as hard to change constructs. Each person supposedly experienced their regular levels of happiness within a certain equilibrium. Further, after every temporary change to this equilibrium, it was believed that people would return back to this level repeatedly (dynamic equilibrium model; Headey and Wearing, 1989). This idea was also supported by genetic studies which found an inherent set-point of happiness in each individual, which acted like a base line, to which they kept returning (Lykken and Tellegen, 1996). It was reported that around $40 \%$ of variability in positive emotionality and $55 \%$ of variability in negative emotionality could be predicted by genetic variations (Tellegen, 1988). Another source of pessimism stemmed from close association of happiness with more or less stable personality traits, such as neuroticism and extraversion (McCrae and Costa, 1990; Watson and Clark, 1997). Similarly, optimistic versus pessimistic attributional styles, regarding an event being internal or external, stable or transient, and/or global or specific, was also documented to be a strong predictor of self-reported happiness (Cheng and Furnham, 2001).

Another set of constraints on happiness were found to be related to socio-demographic factors (such as age, marital status, income). There was a general pessimism about them, since one cannot change these easily. In addition, it was found that even when happiness was influenced by certain changes in life circumstances, it was short-lasting. It seemed that humans quickly adapt/ habituate to any changes in their lives and happiness level. This theory was known as hedonic adaptation (Fredrick and Loewenstein, 1999), or hedonic treadmill (Brickman and Campbell, 1971). 
This brings us to the last set of variables which is about intentional activities. Question abound, whether humans can do certain things to enhance their happiness on a long-lasting basis, and its answers are spread on a broad time-space canvas. This question has been asked in various civilizations, in different time periods of history, by mystics-yogis and philosophers to psychologists. The concepts of an ideal and happy life, ranged from reason (in Greek period) to creative self-expression (in recent times) in the West, and overcoming desires, transcending sense of separation, and living mindfully in the East (Coan 1977). It is reflected in the ideas of eudemonia (Aristotle, 350 BC), of human growth leading towards perfection (Sri Aurobindo, 1948/1999) and of human development culminating in mature and perfect action (Werner, 1926/1957). Psychologist continued this tradition through proposing theories on individuation (Jung, 1933) good mental health (Jahoda, 1958), self-actualization (Maslow, 1968), a fully functioning individual (Roger, 1963) and a healthy mature person (Allport, 1961). The commonalities across these ideas abound, from having a unifying philosophy of life, to the capacity for self-extension and maintaining close interpersonal relationships. Recent work in the field of positive psychology continues this tradition further by scientifically exploring the influences on well-being.

The overall trend indicates two distinct but overlapping perspectives, historically rooted in two separate philosophies namely hedonism and eudaimonia, with different vantage points, such as being subjective versus objective. The hedonic perspective emphasizes pleasure, while eudaimonic perspective focuses on living in accordance with one's deeper self, and self-expression (Waterman, 1993). Research have shown that they are overlapping but different concepts.

\section{The hedonistic perspective}

This perspective follows philosopher Aristuppus ( $4^{\text {th }}$ Century), who proposed that the goal of life is to maximize pleasure and minimize pain, since happiness is the totality of one's hedonic moments. It is similar to the Lokayata/ Charvak school of philosophy in India (one among the nine major schools of classical Indian philosophy). It has remained a controversial idea, never accepted universally. Although in the modern western world, the pursuit of pleasure as a way to achieve satisfaction got wide endorsement. Hedonism is a subjective perspective, since what is pleasurable varies from person to person, and different pleasures may have nothing in common.

The scientific research on pleasure is comparatively recent and sparce. The field expanded with the developments in the study of subjective well-being, especially the hedonic or emotional wellbeing (Diener and Lucas, 1999). When one views emotional well-being as mere physical hedonism, to some it may seem shallow and frivolous, and not worth paying attention to, while doing the serious study of mental life. On the contrary, the concept of happiness goes beyond the idea of simply having fun and recognizes that people are thoughtful beings, who choose what is important in their lives, and pursue those values and goals which may lead to a long-lasting sense of happiness (Diener, Sapyta and Suh, 1998). It was found that emotional well-being can be tapped by various techniques, such as 'savoring' and can also be influenced by engaging in a variety of activities over time (Peterson, Park and Seligman, 2005). Thus, experience of hedonic well-being has some overlap with life satisfaction, and is distinguishable, as well as compatible with the other eudemonic approach to well-being (Kopperud and Vitterso, 2008). It proposed that the chronic happiness level, based on averaging of positive emotions, is more enduring than momentary happiness, while also being somewhat malleable over time, based on the activities people engage in on a regular basis (Lyubomirsky et al., 2005). It was also found that people may vary in their hedonic profiles even with similar happiness levels as captured by the well-being scales, due to 
the variability in affective repertoire or personal judgements of contentment with one's life. Thus, it was considered that, happiness when conceptualized not as a uni-dimensional concept (Argyle, Martin, and Crossland, 1989), but as a multidimensional phenomenon, with components like achievement, satisfaction, enjoyment (Cheng and Furnham, 2003), cooperation, sensuality (Morris, 2004) can point towards the various means of achieving happiness, some of which may represent additional contributions to a happy life.

\section{The eudaimonic perspective}

Fulfilling or realizing one's daimon' (true nature) by engaging in activities that promotes one's highest potentials, is at the core of the eudaimonic view point, which considers happiness is the only 'good' desirable for itself (Nicomachean Ethics, Aristotle, 1985). By exercising one's inherent or acquired abilities, and striving towards realizing them in increasingly better, complex or perfect ways, one can experience joy (Linley and Joseph, 2004). This perspective which takes a more objective position, emphasizes developing virtues (a standard of right or moral excellence), both, the virtues of thought through teaching, and virtues of character through practice/ habit. This approach is associated with the whole person and his/her optimal functioning and development, in terms of exploration of one's interests, enterprise, overcoming of obstacles (Russell, 1930) striving for perfection (Ryan and Deci, 2001), developing inner resources and vitality (Van, Gedrag and Gezondheid, 2005) and engaging with the existential challenges of life.

In modern research, a hierarchical model of strengths and virtues have been proposed (Peterson and Seligman, 2004), where some scholars consider virtues as independent and emphasize developing individual strengths. Others believe virtues are interdependent, that eudaimonic happiness requires all the virtues, and practical wisdom is the master virtue which is essential for solving problems (of specificity, relevance and conflict), that arise whenever strengths must be translated into action in concrete situations (Schwartz and Sharpe, 2006). In general, eudaimonic living is associated with four motivational concepts: (1) being mindful and acting with awareness; (2) behaving in autonomous, volitional or consensual ways; (3) pursuing intrinsic goals and values (e.g. personal growth, relationships, community and health) for their own sake, rather than for extrinsic goals (e.g. wealth, fame, image, power); and (4) satisfying basic psychological needs for competence, relatedness and autonomy, facilitated by the other three concepts (Ryan, Huta and Deci 2008). Thus, instead of outcome, eudaimonic approach focuses on the process of living well.

Research in the field have increasingly found the two approaches, of hedonism and eudaimonia, different, but still overlapping (Selnes, Marthinsen and Vitterso, 2004), leading to the conceptualization of a good life as a multidimensional phenomenon where both are present (King, Eells and Burton 2004; Ryan and Deci, 2001). Hedonism is subjective, and thus is studied by asking people about their sources of happiness. However, in the process it was found that people report life philosophies which are similar to eudaimonia. This is probably due to internalized social norms of eudemonic nature, which then influences their affect and life satisfaction which makes up their hedonic well-being. A few studies have also shown differential temporal association, with hedonic activities leading to immediate happiness, and eudaimonic activities leading to delayed happiness, which increases with repeated efforts and has a spill-over effect on other activities. While hedonic activities are simply pleasure oriented, eudaimonic activities are meaningful and might also involve ego development/ maturity. Association of these two approaches with various other variables like personality, self-esteem etc., have also been found. Waterman (1993) reported strong positive correlation between those activities which are about personal expressiveness 
(eudaimonia), and hedonic enjoyment. There were differences between hedonic and eudemonic approaches too; in terms of (1) opportunities for satisfaction, (2) strength of cognitive-affective components, (3) level of challenges, (4) level of skills, and (5) their importance for the person.

In addition, researchers with an eudaimonic stance have questioned the idea that people always try to enhance their happiness, and avoid unpleasant emotions (are hedonistic in their motivation). Instead, they have suggested that people may be more strategic and sophisticated in managing their moods (Tesser and Rosen, 1975). The eudaimonic perspective on positive emotions is that: (a) being a fully functioning individual is more important than feeling positive per se, therefore under certain conditions, people also need to experience negative emotions (e.g., sadness over loss) (b) studying positive emotions as an outcome of eudaimonic living, and (c) fulfillment of psychological needs may lead to positive emotions (Ryan and Deci, 2001). The dynamics of hedonic versus eudaimonic approach is brought together by the fact that all happy lives may not be good and all good lives may not be happy. Since a hedonically happy life can be an unhealthy one too, the effect of hedonistic living on the number of years lived happily needs to be taken into account (Veenhoven, 2005). The healthy but unhappy life might happen when some important goods in life are either in opposition to hedonism, or require sacrifice of happiness in their service. These goals when attained, are usually associated with heightened happiness. Our happiness level may also serve as a source of feedback regarding our decisions or activities really being an ingredient to a fulfilling life, or not. For example, when one is unhappy in a relationship or at work, it may indicate a need for some change. During difficult times, coping by engaging with existential issues or accommodating with the situations, may lead to maturity and happiness. Thus, a happy, mature person is the most desirable option, as compared to happy but immature person, unhappyimmature person, or unhappy but mature cynical person (King, Eells and Burton, 2004). Therefore, it may be mentioned that different theorists have proposed different pathways towards happiness, broadly divided into eudaimonia and hedonism. However, there is lack of total clarity and convergence of findings as to how these pathways relate to each other and whether they differentially predict affective and cognitive dimensions of well-being.

The eudaimonic approach, given its implications for promotive mental health interventions, will be explored further along with the role of meaning and spirituality for the same.

\section{The eudaimonic approach through meaning and spirituality}

Meaning: Since people quickly adapt to positive events or circumstances, meaningful goals and activities, especially when chosen autonomously can cumulatively and sustainably enhance happiness. Thus, one may say eudaimonic pathway to happiness goes through meaningful activities (Peterson, Park and Seligman, 2005). Meaning can be regarded as one of humanity's tools for imposing stability on life. Essence of meaning is connection, an ability to link two things, even when they are physically separate entities. The famous psychologist Victor Frankl (1959, 1976) is credited with conducting the pioneering research on meaning in life. Meaning has multiple levels; low levels invoke concrete, immediate and specific meaning, while high levels invoke long time spans and broad concepts, such as values and principles (Vallacher and Wegner, 1987). Reker and Wong's model of personal meaning (1988) postulates three components of meaning: cognitive, motivational and affective. The motivational component acts as a partial mediator of influence between the cognitive and the affective component (Halam et al., 1994). Quest for meaning can also be understood in terms of four main needs for meaning, or four patterns of motivation; need for purpose, values, sense of efficacy and self-worth. People who have satisfied all four of these 
are likely to find their lives very meaningful. While there are abundant opportunities to fulfill need for purpose, efficacy and self-worth, the modern world does not offer people a reliable and convincing set of values. There has been a replacement of traditional values with rationality and there is also a loss of consensus about values (Baumister, 1991).

Empirical research supports the relationship of presence of meaning in life, with eudaimonic pathways, self-appraisals and behaviors. On the other hand, search for meaning seems to be related to hedonic self-appraisals and behaviors (Steger, 2006). Although during times of difficulties, the coping mechanism utilized by those low versus high on meaning differs, but low need for meaning in life is not pathological (Desormeaux, 2000). In addition, meaning in life can be influenced by having perspective and being able to see the big picture in life, along with experiencing positive affect which can make people more sensitive to meaning related cues and differentiate meaningful vs meaningless activities (King, Hicks, Krull and Del Gaiso (2006; Hicks and King, 2007). Baumister and Vohs (2005) has also suggested that meaning is necessary but not sufficient for happiness, though majority of times, more meaningful lives will also be happier ones. Further, cognitive appraisal related to positive meaning in specific circumstances can trigger positive emotions, but such appraisals are influenced by personal histories and goals and thus are difficult to control. However, some common mechanism can be utilized, such as reframing or infusing ordinary daily events and activities with positive value, creating or recalling situations that elicit positive meanings (Folkman, 1997; Fredrickson, 2005). Similarly, behaviors related to social and interpersonal relationships and exercise and other physical activities may have potential for meaning infused happiness.

Seligman (2002) had brought these two perspectives, hedonism and eudaimonia together to suggest four different forms of good life. The simplest being 'pleasant life', similar to the hedonic approach, involves successful pursuit of positive emotions associated with present, past and future. The next being 'good life', a more complex approach related to using one's strengths to obtain gratification in the main realms of one's life. It is not a permanent state, but a continuous development of individual strengths and values and leads to authenticity; i.e., being true to one's own character. The 'meaningful life' is closer to optimal functioning and in addition to good life, it is an affiliation to something beyond oneself. It emphasizes using one's strengths and virtues in the service of something much larger, where one is free to conceive the larger aspects of life. Finally, the 'full life' is related to optimum functioning / authentic happiness and builds upon all these three forms. It consists of experiencing positive affect about the past and future, savoring positive feelings from pleasure, deriving gratification from one's signature strengths and using these strengths in the service of something larger than self to obtain meaning (Peterson, Park and Seligman, 2005). Other researchers have also emphasized the need for balance between these pathways, due to the limits of satisfaction which people can derive from a single life domain. It is proposed that involvement in multiple domains would satisfy the full spectrum of human development needs from survival to growth, while fulfillment of only one kind would not lead to high levels of happiness/ SWB (Sirgy and Wu, 2009).

Spirituality: In modern times the term 'spirituality' has been used to denote some kind of subjective religious, mystical or sacred experience, which is private in nature, although no universally accepted definition is found. It overlaps with religion, which also concerns itself with the relationship between humans and Divine, although in a much more organized manner. Further, those who do not believe in organized religions, may still share many of the components of 
spirituality, which in itself is a multi-dimensional concept. Having a personally meaningful framework, deeply committed values, and transformative experiences all are considered to be part of spirituality, and in recent times, with influences from eastern especially Indian tradition and esoteric western influences, the modern spirituality has taken a more psychological approach. Here the psychological ideas related to human potential for growth and meaning are being emphasized, where one continues on the journey towards a non-material, transcendental reality. The other choice is that of spirituality rooted in ethical, moral values, without focusing on the non-material or super-natural.

The relationship between spiritualty and well-being has been discussed and debated. One viewpoint considers spirituality as a key aspect of human health and well-being (WHO, 2001), through offering a meaningful framework in life, given its focus on transcendence and transformation. However empirical research on spirituality and well-being, which is often originating in USA, has found mixed results in terms of both positive and negative association with mental health. This goes with another, more nuanced perspective on spirituality which proposes that having a spiritual life may not be a necessary ingredient of a pleasant, happy life, but it is an important element of living a good life, and thus of authentic long-lasting happiness. Subjective reports of benefits of spirituality abound, and there is now support for a spiritual and compassionate care for the ill. When it comes to religiosity, many large studies often based on national samples, report that well-being is significantly associated with religious certainty (Ellison, 1991), strength of one's relationship with the Divine (Pollner 1989), prayer experiences (Poloma and Pendleton 1991), and devotional and participatory aspects of religiosity (Ellison, Gay, and Glass 1989), even after controlling for age, income and marital status, although the effect sizes are usually not large. Religious behaviors and experiences may offer a sense of meaning in daily life (Pollner, 1989; Steger and Frazier, 2005) and during major life crises (McIntosh, Silver and Wortman, 1993), while also providing avenues for social fulfillment through exposure to networks of like-minded people (Taylor and Chatters, 1988).

While religiosity or spirituality can be a source of meaning and an eudaimonic pathway to wellbeing, it may not always be so. One reason for mixed findings is related to lack of a common definition. It is also important to note that spirituality is multidimensional therefore one needs to look at the specific components amongst the varieties of practices within the broad idea of spirituality. Kees Waaijman has described 4 forms of these practices; Somatic practices, related to depriving the body and diminishing its desires and impulses, and purifying it through fasting etc., (2) Psychological practices, like meditation, (3) Social practices, focusing on others, and interpersonal aspects while reducing ego, (4) practices helping one to focus on divine realization and overcoming an ego centered existence.

Various components of religion and spirituality and factors influencing them, needs to be studied further. In one study it was found that while faith maturity and positive image of God contributed to well-being, negative God-image lowered well-being (Mendonca, Oakes, Ciarrocchi, Sneck and Gillespie, 2007). Even age and race has been found to have differential effect on the relationship between religion, meaning in life and well-being, with older African Americans more likely to find meaning in religion than older White persons (Krause (2003). Possibilities of common underlying mechanisms, such as extraversion, behind association of spirituality and well-being needs ro be further studied.

\section{The cultural criticism of research on spirituality and well-being}


The western ideas of SWB have been criticized on the basis that they ignore cultural perspectives in conceptualizing a good life, that the idea about a 'fully functioning individual' is a culture bound concept (Seligman, 2002) and what is important in one culture may not be equally important in another. In a study with slum dwellers of Calcutta, Biswas-Diener and Diener (2009) found that while they generally experienced a lower sense of life satisfaction than the more affluent comparison groups, they had higher than expected levels of satisfaction, due to the salience of and emphasis on social relationships. This suggests individuals from diverse backgrounds face unique issues that invariably influence their experiences, and what is good for one may not be necessarily good for all. Certain constructs may hold a different cultural meaning and the standards of satisfaction may differ from culture to culture. Even the research literature indicates substantial cultural variations in (1) meanings of happiness (2) motivations underlying happiness and (3) predictors of happiness (Uchida, Norasakkunkit and Kitayama, 2004). Similarly, research on spirituality and well-being has been predominantly situated in an American context. Thus, it is necessary to further explore and examine the magnitude and equivalence of constructs across cultures.

\section{Indian spiritual tradition and happiness}

Spirituality and religiosity related literature often originate from a western context, especially north America, where Christianity is the dominant religion which is closely linked with group religious services, social support etc. On the other hand, in the Indian context majority practice Hinduism, traditionally also known as 'Sanatana dharma'. It is different from the common idea of religion, with diverse conceptualization of Divine, many religious figures, a range of philosophical perspectives, innumerable sacred texts, and a spiritual approach which is lot more personal and individualized and considered to be a 'way of living'. The tradition aims towards individual spiritual experiences, discovery of true self and merging with the universal consciousness. In this context Yoga is a common term used both as one of the many specific schools of philosophies, and also to indicate towards a collections of techniques for spiritual growth (Larson and Bhattacharya, 2016), thus representing a holistic model of spirituality, associated with well-being and happiness. As we will see, there are a variety of such yogic pathways. Further, a variety of happiness has also been discussed in the Indian tradition, using two broad different terms, 'ananda' and 'sukha', depending upon one's psycho-spiritual growth. These will be discussed later.

To understand Indian spiritualty and well-being, it is important to understand its specific perspective on reality, whereby all creation is permeated by Consciousness. It has offered distinct models to understand cosmos and human nature, such as 5 sheaths of existence (panchakosha). As per this model, the human existence is composed of 5 sheaths/ layers; i.e. physical body (annamaya), vital-life force (pranamaya), mental (manomaya), knowledge (vijnanamaya) and bliss (anandamaya) (Taittiréya Upanishad). As a person's identification with one or other of these layers' changes based on his/ her psycho-spiritual development, their quality of happiness too changes (Salagame 2006). The other important model is that of Purusha and prakriti. It originates from the sankhya-yoga framework, whereby the universe is composed of the dual principle of consciousness and matter, the Purusha (pure Consciousness) and the prakriti (primary materiality). The same principle extends to humans, whereby both body and mind are made up of matter (prakriti), while deep within lies the higher Self, or the principle of consciousness (Purusha). This is in contrast to the western conceptualization which considers mind as non-material, origin of consciousness and distinctly different from the body. 
The real purpose of prakriti has been described as two-fold, experience / bhoga and liberation / kaivalya (Pataïjali Yogasütra). When prakriti based body and mind gets reflected in the pure consciousness (Purusha/ inner Self), it starts identifying with thoughts, emotions and actions as its own. It then conceives itself as a separate isolated entity and consequently experiences joys and sorrows (bhoga). On the other hand, the process of separation between prakriti and Purusha is called viyoga, and kaivalya is the state of final, ultimate liberation from prakriti, and achieving union with one's Supreme Self (Purusha). Different traditions of yoga aim towards such liberation from the bondages of prakriti, at least from the lower aspects of prakriti, if not absolute freedom from the prakriti. As per Sri Aurobindo (1999), prakriti is constantly engaged in a process of evolution which he calls 'nature's yoga'. Humans can become aware of such a process and actively contribute towards it, leading to speedier unfolding of this developmental process.

\section{Types of happiness in the Indian tradition}

Ananda: As mentioned earlier, the ultimate aim of traditional Indian spirituality, or Sanatana Dharma is liberation from the phenomenal material reality and experience of one's true self. This state of liberation is supposed to result in ananda - the highest form of blissful happiness and peace. Indian Yogi-philosopher Sri Aurobindo (1999) mentions that there is an 'imperishable Ananda' in everything including the individual soul, with its source in God. It is a 'self-existent bliss' across activities and context and comes from living within. But behind such an ananda is the power of highest God (Puruñottama), to whom people surrender, and who then transforms the dualities of their life (Sri Aurobindo, 1997). Such a spiritual approach, also known as bhakti-yoga, which leads to a connection with the Divine personality and a relationship of 'closest oneness' extends to a feeling of oneness with the entire creation and ananda.

"An entire God-love and adoration extends to a love of the world and all its forms and powers and creatures; in and deep rapture, not the petty ardour of egoistic desire but the ocean of an infinite Ananda." - Sri Aurobindo (1999)

Sukha: It is easy to note that the path of yoga is long and the state of liberation along with constant experience of ananda is not easily achievable for most human beings. Thus, during the long spiritual journey, when the material nature dominates, a person experiences (bhoga) a range, from happiness to misery, based on their level of identification with this material nature. During this journey, happiness which is experienced is termed as sukha. There are further distinctions between 3 types of sukha.

\section{Types of sukha}

As mentioned earlier, since mind is supposedly made up of material nature (prakriti), it is influenced by the 3 qualities or principles (triguna) of nature. Thus, the happiness/ sukha experienced by our mind before it achieves spiritual liberation, is also influenced by them and are of three types. These are further explained below.

Triguna: The 3 gunas are known sattva, rajas and tamas, as discussed in multiple texts such as Chandogya Upanishad, Samkhya karika and Bhagavad Gita. Sattva guna is described as the principle of light, balance, harmony and purity. It is associated with qualities such as cleanliness, truthfulness, dutifulness, detachment, discipline, contentment, determination etc. On the other hand, rajas guna is described as the principle of movement, dynamism and activity and is 
associated with desire, passion, agitation, anxiety, nervousness etc. Finally, tamas guna is the principle of dullness and inertia and is associated with lethargy and in its extreme, even depression (Wolf, 1998). In nature and especially in humans, these guna do not occur in their purity. People are generally a mix of these guna with one or other of these guna dominating at any point of time. Based on these 3 gunas, there are 3 types of happiness/ sukha; i.e., satvika, rajasika and tamasika (Bhagavad Gita). One can consider guna based sukha as a continuum, with sattva and tamas being the two extremes, with rajas in the middle. As the dominant guna changes, corresponding type of sukha emerges.

Satvika sukha: Satvika sukha is about getting satisfaction in higher mind and spirit-based things, leading to fullness and peace. It may be effortful in the beginning, since it requires self-discipline, letting go of habitual pleasures and can also lead to inner churning, however the end result is beneficial. While, Satvika sukha does have an influence of ego and desire, but as a person develops freedom from these it may culminate into ananda (Bhagawad Gita; Sri Aurobindo, 1997).

Rajasika sukha: The rajasika suhka finds pleasure in body, senses, action of its will-power and intelligence. Although it seems desirable at the beginning, it is harmful in long run, due to increasing bitterness related to satiety, disgust or disappointment (Bhagawad Gita, Sri Aurobindo 1997).

Tamasika sukha: The tamasika sukha finds satisfaction in indolence, inertia and ignorance. Bhagawad Gita describes that it results in delusion; both in short and long term.

\section{Pathways to Ananda vs. sukha}

People in general seems to have been somewhat successful in their endeavor to find happiness or 'sukha', since modern research across cultures have reported majority of people experience happiness at any point of time (Diener and Diener, 1996). However, people and cultures differ in their beliefs regarding what experiences are worth having, which kind of happiness one should pursue and how. As discussed earlier, western philosophy and psychology have attempted to answer this question in a specific manner. These ideas often have common elements in terms of a unifying life-philosophy, capacity for self-extension and sustaining intimate relationships. Similarly, in India the focus has been on truth (satyam), ethics and virtues (Shivam), along with beauty (sundaram) (Rao, 2005) and one is also encouraged to cultivate svasthya (state of being 'settled in oneself'), signifying a subjective state of well-being at the levels of body, life-energy (prana) and mind (Sreelakshmi and Shakuntala, 2006). In the Indian tradition, there is a further differentiation between the 3 pathways leading to different types of sukha, i.e., the pleasant (preyas), the good (shreyas) paths and finally the spiritual/ transcendental (nishreyas) path to ultimate bliss (Ananda). The underlying idea is that of a concomitant scope for free-will to choose amongst these paths, by following a certain life-style which may lead to a specific kind of happiness outcome.

Nishreyas, the path to ananda: this is the path which is supposed to lead to liberation and to ananda. It requires going beyond ethical and aesthetic pleasures, cultivating non-attachment (anasakti) and equal-mindedness to outside circumstances (samata), developing self-mastery by yogic practices, discovering one's own inner order (svadharma) and finding meaning, joy and excellence in it. Further it requires uniting with one's inner being (Purusha/ antaratman) and cultivating devotion to Divine personality (bhakti-yoga). Sri Aurobindo (1999) mentions that once 
we connect with the Divine, our inner svabhava along with ananda, naturally unfolds. This ananda is more intense than satvika or even rajasika sukha.

Shreyas, the path to satvika sukha: Satvika sukha is supposedly reached by the path of goodness, in which one considers social and universal well-being. It is a path chosen by the wise (Kaöhopanishad). We may notice this is similar to eudemonic approach, as discussed by Aristotle in Nicomachean Ethics (1985), who emphasized realizing one's true nature (daimon) by engaging in activities that promotes one's highest potentials and living with values. The path of goodness or shreyas, is one which involves balanced pursuit of three puruñartha- natural order (dharma), wealth (artha) and desire (kama) as per laws of Manu (Kuppuswamy, 1985). Further one can reduce tamas, and increase sattva by engaging in penance (tapas), developing deeper knowledge (jïana), having sense control (brahmacarya) and devotion to a higher path (shraddha) (Salagame 2002).

Preyas, the path to Rajsik- tamasika sukha: The pleasant path has been emphasized by Lokayata and Charvaka schools of Indian philosophy, and it supposedly leads to rajasika - tamasika sukha. We may note again, it is similar to hedonistic perspective, originating from 4th century philosopher Aristuppus, focusing on fulfillment of desires, particularly of sensory nature and maximization of pleasure and minimization of pain (Salagame 2002; Mishra, 2019). Although, within rajasikatamasika sukha, there might be sense of fulfillment of sensual desire (tripti) and feeling pleased with an interaction (santosha) (Salagame, 2002), but it is still mixed with ego, thus distorting the ananda. As per Sri Aurobindo (1999), ego's interaction with nature leads to deformation of field (kshetra) and thus one experiences dualities. For example, pleasure-pain is experienced when lower prakriti at prana or senses level deforms soul's ananda, while liking-disliking are experienced when mind deforms the soul's will.

\section{Empirical studies on sukha and mental health}

Given the importance of happiness in human life, the field of psychology has conducted various studies about happiness using modern methods of assessments. These studies define happiness in a manner which fits the definition of 'sukha'. On the other hand, ananda has rarely been researched empirically, due to inherent challenges in studying it, such as finding study participants who have experienced ananda, its measurement etc. The current author has been involved in a series of studies exploring happiness and triguna in educated urban Indian adults, using a mix of methodologies. These studies have given deeper insights, as well as validated some of the ideas from ancient Indian tradition.

Experience of sukha: The popular way to measure happiness in the field of psychology is selfreport of frequent experiences of positive emotions, infrequent experiences of negative emotions and generally feeling satisfied with one's life. In one of our studies, it was found that majority of people in urban Bangalore were happy on these measures (Agrawal et al. 2010). This result was similar to those found in other studies across the world, and thus it may be considered that at any point of time most people across cultures (Diener and Diener, 1996), including India (Agrawal et al. 2010) do experience sukha.

Triguna and sukha: Since currently there are no measures available to directly assess satvika or rajasika-tamasika kind of happiness, one needs to study them indirectly by analyzing the correlation between triguna and happiness. Through this method we found that sattva was 
associated with happiness. But both rajas and tamas were related to unhappiness, indicated by a significant correlation with a higher tendency to experience negative emotions (neuroticism) and having low life satisfaction (Chandana, Agrawal, Sharma and Murthy, 2020).

Similarly, in another study it was found that higher levels of satvika quality of anasakti was associated with happiness, while a higher level of ego (ahamkara) whereby rajas is dominant, was associated with both frequent positive and negative emotions. This indicates that while sattva is predominantly associated with happiness, higher levels of rajas may be associated with a life full of roller-coaster of emotions, with experience of both happiness and unhappiness (Gupta and Agrawal, 2020).

Pathways to sukha: No exact measures have been developed with respect to the shreyas, preyas and nishreyas pathways; therefore, one has to use other measures which may cover the construct described under these pathways as closely as possible. Like ananda assessing its related path of nishreyas may be difficult, however since Indian concept of preyas and shreyas can be mapped on to the Greek concept of hedonic and eudaimonic pathways respectively, tools to assess them may be used to assess these Indian constructs. When people were asked about their preferences for happiness, they seemed to choose a mix of activities to experience happiness, and these activities fit the definition of either hedonic (preyas) or eudaimonic (shreyas) kind of pathway. The choice of these activities was dependent on the context and situation but on an average, people prefer those activities which might be categorized as eudaimonic or shreyas pathway, whereby they give importance to relationships, meaningful activities, duties and living with values. It was also found that taking a shreyas pathway (with importance given to meaningful framework and eudemonic activities) was associated with happiness. On the other hand, preyas kind of pathway, with frequent engagement in hedonistic activities and ignoring meaningful eudemonic activities was associated with negative emotions (Agrawal, Murthy, Mehrotra, 2010). This may somewhat validate the description of rajasika sukha (as decribed in Bhagavad Gita), which is supposed to be pleasant like nectar in the short term, but is harmful like a poison in the long term.

Use of alcohol may be considered as one of the common hedonistic (preyas) activities, even to a harmful extent, in modern times. When a person gets dependent upon alcohol and is unable to stop oneself, it is diagnosed as alcohol dependence. In another study with participants diagnosed with alcohol dependence, it was found that this group had higher levels of tamas. They also had lower sattva, lower positive emotions and higher negative emotions, as compared to people who were not dependent on alcohol (Chandana, Agrawal, Sharma and Murthy, 2018). This finding too was

on similar lines as described in Bhagavad Gita, where tamasika sukha is harmful in the beginning as well as in the long term. Thus, one may hypothesize that types of sukha may be associated with mental health and rajsik- tamasika sukha may be related to poor mental health or even clinical conditions while satvika sukha may be associated with positive mental health.

\section{Yoga: A comprehensive psycho-spiritual pathway to deeper happiness}

In the Encyclopedia of Indian Philosophies (Vol 12), Larson and Bhattacharya (2016) mention that the term Yoga is often used for all those techniques which people practice to find release from their existential suffering and achieve unity consciousness. In this sense Yoga may be considered as an applied physical-psycho-spiritual pathway (Nishreyas) rooted in the Indian civilization, for finding highest state of existence and bliss (ananda). The yoga tradition is vast, with diverse living tributaries of knowledge systems (oral and textual). It has grown and survived various challenges, 
evolved within a system of training under a Guru (spiritual teacher), supported by one's experimentations, observations and reflections - contemplations, and refined by sharing of experiences and debates-discussions. The 'time-space framework' is wide, and it is established inside a network of personal, interpersonal, community and cosmic web (Menon, 2005; Dalal and Mishra, 2010)

Recent research has also found convincing evidence to suggest that Patanjali's 'Astang Yoga' works like an integrative self-regulation module, with both top- down and bottom -up components (Gard, Noggle, Park, Vago and Wilson, 2014; Schmalz, Powers and Blom, 2015; Pascoe and Bauer, 2015; Payne and Crane-Godreau, 2015). Other psychological mechanisms such as meta cognition, disidentification, deconditioning and introceptive awareness might be behind beneficial effect of yoga on mental health (Vorkapic, 2016). Majority of yoga research has been dominated by hatha-yoga (Yang, Bernardo, Sereika, Conroy, Balk, et al. 2009), which covers mainly the yogasana (physical postures) aspect of Patanjali's 8-fold path (Gard et al. 2014) ignoring the psycho-spiritual aspects of Yoga, which has emphasized certain attitudes, cognitions and emotions. Although more and more studies are integrating yoga with various types of meditation (Uebelacker, Epstein-Lubow and Gaudiano, 2010; Mendelson, Greenberg, Dariotis, Gould, Rhoades, et al. 2010) and/ or pranayama, the breath related practices (Pullen, Thompson, Benardot, Brandon, Mehta, et al. 2010). There are many psychological ideas in the Yoga tradition directly related to beliefs, attitudes, cognitions, emotions and life choices and aalthough some of these have been researched individually, they have rarely been brought together in empirical research.

While research body has predominantly focused on the therapeutic effect of Yoga, a few studies did explore well-being and positive mental health outcomes of yoga in community population (Chung, Brooks, Rai, Balk and Rai, 2012; Noggle, Steiner, Minami and Khalsa, 2012; EastmanMueller, Wilson, Jung, Kimura and Tarrant, 2013). In a meta-analysis, Knobben (2013) found few research studies testing the effect of yoga on well-being, with large impact on emotional wellbeing, and medium for both psychological and social well-being. Recently, two separate reviews have also reported publication of newer studies exploring yoga and positive mental health, in terms of psychological well-being, emotional well-being, life satisfaction, social relationships (social well-being), empathy, mindfulness, self-compassion and resilience. But these results seem to indicate weak positive relationship between yoga and positive mental health (Hendriks, deJong and Cramer, 2017; Domingues, 2018). Such weak results might be explained in the light of not having any direct intervention pathway in these studies related to cognitions, emotions, life choices etc. based on yogic and Indian psychological traditions.

Salagame (2011), in his review of Bhagavad Gita, Patanjali's Yoga Sutra, and Yoga Vasishta found various approaches given in these texts, which may be useful for mental health and wellbeing. This includes developing a witness stance, self-discipline, detachment, certain motivational and attitudinal orientations, acceptance and compassion. Similarly, Bhavnani (2014) has discussed various dimensions of Yoga, along with a variety of therapeutic skills based in yogic philosophy, such as swadhyaya, pranayam, pratyahara, dharna, dhyana, bhajana, vairagya, chittaprasadanam, maitri, mudita, karuna, upeksha, trataka, pratipakhsa bhawana etc. Some of these ideas may be usefully brought together to develop well-being programs. Thus, a need was felt to develop a model based on core yogic texts, for the purpose of future well-being interventions. If such a model is present, it may theoretically guide various promotive mental health interventions, which in turn 
may benefit anyone on the mental health continuum (Keys and Lopez, 2002; WHO 2004), from illness to wellness, from a broad yogic spiritualty stance.

\section{Analysis of core yogic texts}

To develop a yoga-based psycho-spiritual model, thematic analysis of three yoga texts; Bhagawad Gita, Patanjali Yoga Sutra and Yoga Vasistha was conducted. These texts are some of the most important and may be considered core yoga texts (Salagame, 2011) The process started with multiple readings of these texts leading to individual themes, which were then covered under several overarching broad themes.

The texts: Bhagawad Gita, translated as 'The song of Divine' in English, is part of a larger epic Mahabharata written by Sage Veda Vyasa in ancient India. It also stands as the best-known Hindu scripture encompassing 700 verses, synthesising the three major paths of yoga; i.e., bhakti (devotion), jnana (knowledge) and karma (action). Gita, a conversation between Lord Krishna and his friend and mentee Arjuna, has inspired generations of philosophers and mystics, from Emerson to Sri Aurobindo. The Yoga Vasishta, written by Sage Valmiki has two versions, the larger with 29,000 verses and a smaller one used for this study with 6,000 verses. It captures the conversation between Lord Rama and a group of sages lead by Sage Vasistha. The Patanjali Yoga Sutra is a compilation of 196 aphorisms (sutra) by Sage Patanjali, covering the theory and practice of Yoga in four short chapters (pad). Here the focus is on compilation of steps for concentration and resulting inner experiences, and in the process documenting the helpful and unhelpful factors.

In general, these texts have given a practical approach to spirituality, which anyone can try for themselves. Two out of these three texts have utilized a narrative framework, with dialogue between a 'main character/hero' and a mentor (guru) figure (Bhagawad Gita and yoga Vashishtha). The narrative begins with the 'hero' having a psychological crisis, matching the modern description of anxiety/ depression. Subsequently he is helped ('talking cure') by the mentor, through clarifying doubts and teaching a variety of applied techniques (e.g., variety of meditations, breath control/ pranayama, as well as cultivation of specific virtues and skills). In Gita, amidst a background of war, the hero Arjuna reports symptoms similar to anxiety, and in Yoga Vashishtha it is Lord Rama, who forgetful of his Divine status, is experiencing symptoms which may be termed as an existential Depression. The third text (Patanjali YogaSutra) is briefer, without a narrative, and focuses on steps for deepest meditative states/ Samadhi, resulting inner experiences and psychic powers. It also discusses states of mind, causes for suffering and helpful and unhelpful factors for meditation. Based on the themes from these 3 core texts, a model was developed. These themes are interconnected and each one of them interacts with the other to provide insight.

\section{The Yogic model}

As mentioned earlier, this interlinked model was developed based on thematic analysis of core yogic texts. As we will note that each of the component is also related to the other components, and makes a whole framework, which may be utilised in part or full for well-being interventions. However, one needs to remember these components are primarily focused on spiritual growth and positive mental health is merely a by-product. 
Perspective: One of the core components of this model is about yogic perspective on life and universe, which includes an assumption related to the purpose of creation and its principle components. The overarching perspective is related to the inherent unity of Consciousness. It considers Consciousness, together with primordial materiality (prakriti) has led to the diverse creation in the universe. And thus, creation is considered to be an interaction between matter and Consciousness, and Consciousness is permeated in everything around us. The material component is influenced by the 3 qualities (triguna), i.e., tamas/ inertia, rajas/ dynamism and sattva/ lightharmony. Thus the quality of this diversified existence is in a way determined by the proportion or dominance of these three gunas. The resulting creation is considered to be cyclic and also ever changing. Human existence is also an interaction of both matter and Consciousness. This consciousness or deeper Self is distinct from mind, which is multi-layered and complex, but merely reflects the Consciousness. Mind is subtle matter, is influenced by triguna and thereby leads to varied experiences for humans.

The main cause of suffering, as per the yogic perspective is ignorance of our deeper Consciousness or true being, although multiple overlapping models have been suggested to explain suffering (types of cover on this Consciousness, types of suffering, types of mental states etc.). And overcoming this suffering requires recognition of deeper Self, by achieving some sense of separation from the material aspects (Viyoga). This may be done even while pursuing the entire range of human aspirations (purushartha; right-ethical action, wealth, pleasure \& existential freedom) based on personal conception. The perspective also takes into account the different kinds of happiness and pathways towards them. It is an experiential journey, with growth across births, thus no effort on this yogic spiritual path is lost.

Self: In the yogic tradition, two kinds of 'self' have been considered, an outer self and an inner self. The outer or lower self is the ego sense, identified with the body. The metaphor of a pot, (or a house) is used to denote how this outer self is just a protective cover for the inner space. Outer self is limited by a sense of separation from others, and the larger world. It thrives on having a sense of attachment, possession and ownership related to people, things, ideas and experiences. It also mistakenly thinks of itself as being the master of one's world. This outer or lower self can be like an enemy or friend. When one is on a journey of spiritual growth and the discovery of deeper self, this outer self needs to be transcended.

The deeper self is also considered as one's higher self, and is described as the unaffected witness within, identified with the entire universe and being the spark of divinity in humans. While the body dies, the deeper self is imperishable. It is the center of our individuality and is also called 'Mahatattva'.

The separation from outer and deeper self, through the means of spiritual development is also knows as 'Viyoga'. This process starts with acknowledging the possibility that there is a deeper Self to be discovered. Freedom from ego can come gradually by self-observation and self-analysis, where one holds one's behavior 'at a distance'. One can know about one's limitations, thereby reducing pride and giving up desires, anger, agitation, I-ness/ my-ness. At cognitive level, notions of 'I am...' and 'this ... is pleasure', leads to ego, desire and subsequent bondage. Therefore, one can deconstruct one's sense of ego-self by contemplating on 'I am not ...', and confronting this is pleasure, with thoughts of 'this ... is not pleasure'. Further, contemplating on the nature of cosmic Consciousness, on certain deeper-self based affirmations would be steps on the path. Surrender to 
Divine and finally renouncing this idea of being a 'renouncer', may lead to the highest experience of ego less state, where only the subject alone exists.

Divine: A variety of forms and descriptions have been used for the Supreme reality/ One Consciousness / God. For example, in the universe whatever is glorious, beautiful, mighty etc., is just a reflection of this Supreme power, who is still not limited by these, or a variety of forms have been described, each of which emphasizes a different, though essential qualities in the creation (e.g. knowledge, freedom, forgiveness, truth, self-control etc.). This power is supposedly present in different states, such as involved with the creation, uninvolved witness of all movements in the creation, and in a transcendent state encompassing other two states and beyond. The Divine is also described as actor, as well as the enjoyer of the cosmic play, witness and also the one who gives sanction to it and also sustains the happenings in the world. The Divine is still free from all human limitations of actions and its outcomes. This supreme power is also believed to be allcompassionate, for everyone, without differentiation (e.g., for the titan and the oppressed), but also acts with love, through people based on their qualities/ abilities.

The yoga of bhakti is the chosen path here, in which one tries to develop a secure attachment with one's preferred God. This is done through seeking of the Divine, by love-adoration, by finding safety in and worship of the Supreme, and by remembering God especially in critical moments. It is also associated with developing self-awareness, knowing one's limitations, evolving psychologically-ethically, following a divine life and doing everything as an offering to the God. This may culminate in finally abandoning all rules and taking refuge in Divine and surrendering to Divine.

Other: Since there is one Consciousness behind all the multitude of creations, there is an inherent connection between everything and everyone. Discovering such oneness (sense of Divine in all) is a process and also an outcome of the spiritual path. This has to be done by following Social-ethical codes of non-harming, truthfulness, non-stealing, sense-control and non-greediness (Yama). Further, one needs to cultivate the interpersonal attitudes of harmony and universal goodwill, such as loving kindness and friendliness (maitri), appreciative joy (mudita), compassion (karuna) and equanimity (upeksha). One not only develops discipline of speech, in terms of soft-pleasant and truthful speech, but also of working towards social welfare (lokasamgraha).

Action: The terms 'karma' has been used to denote creative movements in yoga. The right karma is that action, which allows the expression of one's inner nature. All actions and their outcomes are supposedly influenced by many variables (e.g., causes of action, its types etc.) which interacts with a variety of motivations behind the actions and the operations of the universal forces (e.g., the principles of trigunas). When ones' action is based on one's inherent nature and context (such as at the right time), and when one acts with persistence while being devoted to a higher cause, it leads to liberation from bondages of karma. Such actions become 'dharma' or the right action and lead to the greatest good and subsequently to authentic happiness. It has also been mentioned that such excellence in action leads to growth or 'rising upwards'.

In this model, the emphasis is on finding one's inner nature (svabhava), one's right action (swadharma) and working for the well-being of everyone (lokasangraha). One needs to discover and choose the right action for oneself, based upon one's inner nature and context. One also needs to focus on work at hand, and not get troubled by the outcomes or its thoughts, since one has 'right to action, not to its fruits'. Another important idea here is that of 'sacrifice', where one offers one's 
action to the universal forces, which supposedly brings the results, and thereby a cycle of interchange between God and man occurs. One's actions can be done with equality of will and in harmony with the Divine will (not working for one's desire and ego). One can start with renouncing the fruits of action, go on to do everything for the sake of Divine as one's offering. Working as an instrument in the attitude of karma-yoga, and finally with faith and surrender help reach the state of complete union with Divine. Before that stage comes, one can try to work in a self-aware and selfless manner, by renouncing the claims of doership, and with focus, firmness, excellence, doubtlessness, discretion, in silence, purity, non-violence and self-control. There is emphasis on working with the right attitude and principle, like a witness, holding away, balanced, without desire and expectations, without like-dislike, jealousy, and by removing dependency and attachment for good-bad outcomes. Freedom in inner contact, along with equality and acceptance for whatever outcomes come in one's way, and knowing that behind everything is the equal witness Self, is encouraged. This is known as 'mahakarta'.

Experience: A model has been offered in yogic texts, where identification with one's actions and experiences lead to a conditional mental consciousness, in both wise and unwise people. Such conditioning of mind in turn creates desire for similar experiences, which leads to movement of prana (life-force / breath) along with attachment/ clinginess to a mental idea or fantasy. This works like a seed for the mental tree and in turn may create more thoughts, feelings and actions. In addition, when we identify with such movements in mind and that of prana, it builds our ego. The ideal state is that of equality in varied experiences (sama), when one doesn't hate or yearns for anything and enjoy all the natural experiences of life. This happens when the inner self (soul) remains a witness to happenings of the three gunas, without making arbitrary distinctions, and without getting affected by the dichotomies of pleasure-pain, fortune-misfortune etc. It enjoys the playful engagement in natural action, by developing non-attachment. Such state leads to a sense of freedom. In this state only rasa (savoring/taste) from varied experiences remains and even that ceases when the Supreme is seen or experienced.

To develop this state one needs to develop a witness attitude (sakshi bhava), observing the play of gunas, along with turning one's senses and attention inwards (pratyahara, through techniques such as trataka, japa, nada yoga, kirtan etc.) and inner yoga (antaranga; dharana, dhyana, Samadhi, together with samyama). Yoga practice along with enquiry is required. One needs to recognize that the craving for pleasure is never fulfilled since desire never ends and contemplate on cultivating dispassion. Similarly, there is need to regulate the breath since it signifies the movement of life force, by enquiry of cause of movement, practice of pranayama, by sense control, and self-discipline. A variety of practices from hatha yoga, such as bahirang yoga, mudra, etc. have also been suggested. Self-observation and contemplation (swadhyaya), surrender, abandoning all notion (sankalpa) have been emphasized. Another powerful technique is that of offering as sacrifice one's sense experiences, sense objects, vital force etc. Other techniques are related to focusing on opposite thoughts and feelings especially when mind is disturbed, sometimes removing one's attachments in a sudden action, and cultivating positive interpersonal qualities. The texts discuss in details about giving up craving, desire, likes and aversions, lust, anger, grief, delusion, greed, violence etc., since it will decrease the bondage of the phenomenal world. The spiritual tapas / heat of such efforts will supposedly burn the conditionings on the mind (samskara) and unfold higher consciousness and powers. Given the importance of experiences and emotions, the texts further go in details of other ways to cultivate even mindedness or samata, such as engaging in austerities, study of spiritual texts, gaining knowledge, cultivating focus through 
concentration, focus on a passionless person, sleep yoga, and other kinds of meditation on preferred objects (by rituals, pooja, chanting, kirtan, prayer, deity, enquiry etc.). It also emphasizes personal discipline (niyama), silencing the mind (antarmaouna), self-analysis and self-discipline. Disengaging one's identity from experience, going beyond dualities, increasing the quality of sattva and decreasing rajas-tamas, not being agitated or make others agitated and developing equality in varied experiences are other suggested practices. Engaging in virtuous, meritorious acts and being solitary, decreasing one's material possessions, deconstructing one's ego, selfpurification will also lead to decreased sorrow, and direct knowledge. This path will lead to state of 'Mahabhokta'.

Positive qualities: In the path of growth, development of a variety of positive qualities and values have been emphasized, such as harmonious relationships (e.g. friendliness without attachment, goodness, magnanimity, gentleness, aversion to fault finding, not make others agitated, honesty, non-violence, forgiveness, not having any ill-will, along with veneration of teacher) a certain approach to life (courage, peacefulness, wisdom, fearlessness, steadiness, equanimity, not getting agitated, equality, truthfulness, steadfastness, self-control, rising above discord and finding the harmonizing principle, desirelessness, purity, absence of perversion, endurance, intelligence, contentment, straightforward, doing the right things, goodness etc.). These positive qualities have been variously termed as divine qualities or daivik or satvik qualities.

\section{Discussion and conclusion}

The paper discusses parallel ideas on happiness and pathways to happiness in modern western literature and ancient Indian tradition. The western literature has proposed subjective versus objective happiness and hedonic versus eudaimonic pathways. The Indian tradition has discussed a range of happiness from ananda to three types of sukha. Although few are able to take the path of ananda, many more can be encouraged to gradually move from rajsik- tamasika to satvika sukha, by choosing the difficult but good path of shreyas. Empirical studies from both the traditions have been presented. Modern research has shown that, although more than half of our happiness is tied to genes and circumstances, however there is still much scope for intentional activities that can change our level of happiness. When people take the eudaimonic path or that of shreyas to find satvika happiness, it may need deliberate effort, they may have to gradually let go of many of their desires and ego needs and balance it with concern for others. It may also lead to inner struggle and difficulties related to managing outer changes, but in the end it may be worthwhile. On the other hand, rajasika-tamasika sukha may seem attractive at the beginning, but will lead to more unhappiness later and may even be unhealthy. Thus, we need to follow the rule that 'prevention is better than cure' and develop mental health programs to promote satvika sukha and carry such training programs in schools, colleges, work-places and other community organizations. This will also lead to prevention of unhappiness and mental health problems due to choosing rajasikatamasika sukha. The Yogic model presented may be used to develop promotive mental health interventions based on Indian psychology.

\section{Recommendations}

Since there are interesting parallels between western and Indian models of happiness, the Indian model also needs to be given attention.

The relationship between various types of happiness and mental health and well-bring variables need more studies. 
Given that yoga based empirical research has ignored the psychological aspects, the yogic psychospiritual model proposed in this chapter needs to be tested as an intervention.

Long-term yoga practitioners need to be studied qualitatively for psychologically significant changes and may even be followed up for many years to get deeper insights into some of the phenomenon described in the yogic texts.

Undertaking: This paper is an original work and has not been published earlier.

Acknowledgement: The author would like to express appreciation to Dr. Sushrutha for his valuable inputs to portions of this manuscript.

\section{Selected references:}

Agrawal J., Murthy P. \& Mehrotra S. "Personal Meanings of Happiness and Positive Affect Regulation in Daily Life". PhD diss., NIMHANS, Bangalore, (2010).

Agrawal, J., Murthy, P., Philip, M., Mehrotra, S., Thennarasu,K., John,J., Girish,N., Thippeswamy, V. \& Isaac., M. "Socio-demographic Correlates of Subjective Well-being in Urban India," Social Indicators Research: An International and Interdisciplinary Journal for Quality-ofLife Measurement, 101, no. 3 (2011): 419-434. https://doi.org/10.1007/s11205-010-9669-5 Aurobindo, Sri. Essays on Gita. Pondicherry: Sri Aurobindo Ashram Publication Dept, (1997). Aurobindo, Sri. The Synthesis of Yoga. Pondicherry: Sri Aurobindo Ashram Publication Dept, (1999).

Chandana N., Agrawal J., Sharma M.P. \& Murthy P. "Triguna, Anasakti, Personality and Subjective Wellbeing: A Comparison of Healthy Males with Males Having Alcohol Dependence." Unpublished manuscript, 2020.

Diener, E., \& Diener.C., "Most People Are Happy,” Psychological Science, 7, no. 3 (1996): 181185. https://doi.org/10.1111/j.1467-9280.1996.tb00354.x

Gupta K., Singh S. \& Agrawal J. "A study of psychological processes and outcomes of ahamkara and ansakti." Unpublished manuscript, April (2019).

Kuppuswamy, B. Elements of Ancient Indian Psychology. New Delhi: Vikas Publishing House, (1985).

Mishra, S. "Two Paths: Shreyas and Preyas." Bhagavadgita (blog). (25 March, 2018) http://bhagavadgita.org.in/Blogs/5ab0b9b75369ed21c4c74c01

Patanjali. Patanjali YogaSutra. The science of Yoga. Translated by Iqbal Kishen Taimini. Chennai: The Theosophical Publishing House, (2001).

Rao, K.R. "Scope and Substance of Indian Psychology" in Towards A Spiritual Psychology: Essays in Indian Psychology, edited by K.R. Rao and S.B. Marwaha, 18-40. New Delhi: Samvad India Foundation (2005).

Salagame, K.K. Psychology of Meditation: A Contextual Approach. New Delhi: Concept Publishing Company, (2002).

Salagame, K.K. "Health and Well-Being in Indian Tradition," Psychological Studies, 51, no 4 (2006).

Sreelakshmi, R., \& Shakuntala, M.N. "Psycho-Physiological Concept of Health and Nutrition: Qualitative Vibration of Food," in special Issue, Psychology of Health and Well-Being, Psychological Studies, 51, no. 2-3 (2006): 187-194. 
Wolf, D.B., "The Vedic Personality Inventory: A Study of the Gunas," Journal of Indian Psychology, 16 (1998): 26-43. 